\title{
Paeoniflorin blocks the proliferation of vascular smooth muscle cells induced by platelet-derived growth factor-BB through ROS mediated ERK1/2 and p38 signaling pathways
}

\author{
XIANWEI FAN, JINTAO WU, HAITAO YANG, LIJIE YAN and SHANLING WANG \\ Department of Cardiology, Henan Provincial People's Hospital (Zhengzhou University \\ People's Hospital), Zhengzhou, Henan 450003, P.R. China
}

Received June 27, 2016; Accepted July 14, 2017

DOI: $10.3892 / \mathrm{mmr} .2017 .8093$

\begin{abstract}
The proliferation of vascular smooth muscle cells (VSMCs) contributes to the development of vascular remodeling. In the present study, the effect of paeoniflorin (PAE) on the platelet derived growth factor-BB (PDGF-BB)-induced proliferation of primary cultured rat VSMCs and its molecular mechanism was investigated. The toxicity was determined by the try pan blue exclusion test. Cell proliferation was determined using a CCK-8 assay, DNA synthesis was assessed by measuring the incorporation of BrdU. Cell cycle progression was determined using PI staining and fluorescence-activated cell sorting. The level of intracellular reactive oxygen species (ROS) generation was assessed using dichlorodihydro fluorescein diacetate. mRNA expression was determined by reverse transcription quantitative polymerase chain reaction. Changes of p38, JNK, ERK1/2 signaling pathways were determined by western blot analysis. Cell migration was detected by scratch assay. PAE was demonstrated to significantly inhibit VSMC proliferation induced by PDGF-BB in a dose-and time-dependent manner without cell cytotoxicity. Thus, PAE blocked progression through the G0/G1 to Sphase of the cell cycle. Furthermore, inhibition of the cell cycle was associated with
\end{abstract}

Correspondence to: Professor Shanling Wang, Department of Cardiology, Henan Provincial People's Hospital (Zhengzhou University People's Hospital), 7 Weiwu Road, Zhengzhou, Henan 450003, P.R. China

E-mail: wangshanling321@126.com

Abbreviations: CCK-8, Cell Counting Kit-8; CDK, cyclin-dependent kinase; CDKN1A, cyclin-dependent kinase inhibitor 1A; ERK, extra cellular signal-regulated kinase; FACS, fluorescence-activated cell sorting; FBS, fetal bovine serum; JNK, c-Jun N-terminal kinase; MAPK, mitogen-activated protein kinase; OPN, osteopontin; PAE, paeoniflorin; PDGF-BB, platelet derived growth factor-BB; ROS, reactive oxygen species; VSMCs, vascular smooth muscle cells

Key words: paeoniflorin, proliferation, vascular smooth muscle cells, mitogen activated protein kinases the inhibition of them RNA expression of cyclin D1, cyclin E, cyclin dependent kinase (CDK) 4 and CDK2 as well as with increased cyclin dependent kinase inhibitor 1A mRNA expression in PDGF-BB-stimulated VSMCs. Further studies showed that the beneficial effect of PAE on blocking VSMCs proliferation was related to the suppression of the ROS-mediated extra cellular signal-regulated kinase (ERK)1/2 and p38 signaling pathways, although PAE had no significant effect on the c-Jun $\mathrm{N}$-terminal kinase signalling pathway. These results demonstrated that PAE suppressed PDGF-BB-induced VSMC proliferation through the ROS-mediated ERK1/2 and p38 signaling pathways, suggesting that it may be a feasible therapy for vascular remodelling diseases.

\section{Introduction}

When the internal environment of blood vessels is in disorder, endothelial cells produce a large amount of reactive oxygen species (ROS) through the NADPH oxidase pathway, there by inducing the abnormal proliferation of vascular smooth muscle cells (VSMCs), platelet accumulation and vascular reconstruction (1). VSMC proliferation and migration are important in vascular reconstruction; they cause intimal thickening and consequently atherosclerosis and restenosis, following percutaneous coronary intervention or venous transplantation $(2,3)$. Therefore, inhibiting the abnormal proliferation of VSMCs is instrument altoreversing vascular reconstruction. However, specific drugs against the proliferation of VSMCs have not emerged yet. Developing such drugs with minimal toxicity and side effects could significantly contribute to improving vascular reconstruction, life quality, and survival of patients with coronary artery diseases.

Incases of vascular injury, platelet-derived growth factor-BB (PDGF-BB) is released. Its binding with cell membrane receptor PDGFR- $\beta$ activates NAPDH oxidase to produce large amounts of ROS, which induce the activation of various down stream signalling pathways and mitogen-activated protein kinase (MAPK) signalling pathways $(4,5)$. In VSMCs, the activation of MAPK signalling pathways such as the extracellular signal-related protein kinases 1 and 2 (ERK1/2) pathway and p38 MAPK pathway can lead to cell proliferation and migration as well as the change of VSMCs 
from the contractile phenotype to the proliferative phenotype (6). Lee et al (7) demonstrated that reduced ROS inhibits PDGF-BB-induced proliferation and migration of VSMCs.

Paeoniflorin (PAE) is a monoterpene glycoside from Paeonia lactiflora that reputedly performs various biological functions, including anti oxidative, anti-free radical, anti platelet functions, and improves micro circulation and immune regulatory effects (8). In studies on cardio vascular diseases, PAE has been demonstrated to improve myocardial infarction (9), LPS-induced myocarditis (10) and myocardial ischemia/reperfusion injury in rats (11), by inhibiting inflammation. PAE can also improve pressure overload-induced cardiac remodeling by inhibiting transforming growth factor (TGF)- $\beta /$ Smads and NF- $\kappa$ B signalling pathways (8). Never the less, whether PAE has a therapeutic effect on VSMC proliferation and migration in vitro induced by PDGF-BB, as well as its effect on MAPK signalling pathway, is still unknown. In the present study, in vitro experiments were performed to examine whether PAE possessed protective effects, and the potential mechanism was discussed.

\section{Materials and methods}

Materials. PAE (99\% as determined by high-performance liquid chromatography analysis) was ordered from Shanghai Winherb Medical Technology Co., Ltd. (Shanghai, China). Recombinant human PDGF-BB was purchased from Pepro Tech, Inc. (Rock Hill, NJ, USA). Cell Counting Kit-8 (CCK-8) was obtained from Dojindo Molecular Technologies, Inc. (Kumamoto, Japan). A cell proliferation ELISA, BrdU (colorimetric) kit was purchased from Roche (11647229001; Roche Diagnostics, Basel, Switzerland). Propidium iodide (PI) was purchased from Sigma-Aldrich; Merck KGaA (Darmstadt, Germany). TRIzol was purchased from Invitrogen; Thermo Fisher Scientific, Inc. (Waltham, MA, USA). Primary anti bodies to recognize the total and phosphorylated (p-) forms of p38 (p-p38, 4511P; p38, 9212P), ERK1/2 (p-ERK1/2, 4370p; ERK1/2, 4695), c-Jun N-terminal kinase (JNK) (p-JNK, 4668P; JNK, 9258) and GAPDH (2118) were ordered from Cell Signaling Technology, Inc. (Danvers, MA, USA). Membranes were incubated with the secondary antibody goat anti-rabbit immunoglobulin G (926-32211; LI-COR Biosciences, Lincoln, NE, USA). Sprague-Dawley (SD) rats (150-200 g) were ordered from the Zhengzhou University Center for Animal Experiment (Zhengzhou, China). For the in vitro study, PAE was dissolved in DMSO (Sigma-Aldrich; Merck KGaA).

Cell culture. VSMCs were collected from the thoracic artery of male SD rats aged $<8$ weeks. All the animal experiment procedures conformed to the Guidelines for Animal Care and Use, and the protocol was approved by the Animal Care and Use Center of the Zhengzhou University People's Hospital (Henan Province People's Hospital; Zhengzhou, China). The cells were cultured and prepared at $37^{\circ} \mathrm{C}$ in a $5 \% \mathrm{CO}_{2}$ incubator, as described previously (12). The culture medium consisted of Dulbecco's modified Eagle's medium (DMEM) (11330032; Gibco; Thermo Fisher Scientific, Inc.) supplemented 1:1 with F-12 nutrient mixture F-12 + 10\% fetal bovine serum (10099; Gibco; Thermo Fisher Scientific, Inc.) $+1 \%$ penicillin and streptomycin. When the cells covered $80 \%$ of the bottom of the culture flask $\left(2 \times 10^{5}\right.$ cells $\left./ \mathrm{ml}\right)$, cell passage was performed by digestion with $0.25 \%$ trypsin. VSMCs of the third to fifth generation were used. Prior to experiments, the cells were starved by culturing them in DMEM/F12 containing $0.5 \%$ FBS and $0.5 \%$ penicillin and streptomycin (FBS can partially counteract the activity of antibiotics) for $24 \mathrm{~h}$ to synchronize the cells. Cells were pre treated with different concentrations of PAE for $1 \mathrm{~h}$ prior to stimulation with PDGF-BB (20 ng/ml).

Cell viability. The toxicity of PAE on VSMCs was determined by the trypan blue exclusion test. After 12, 24 and $48 \mathrm{~h}$ incubation with PAE of different concentrations $(50,100,200 \mu \mathrm{M})$, the VSMCs were removed from culture and the cells that excluded $0.4 \%$ trypan blue $\left(37^{\circ} \mathrm{C}\right.$ for $\left.3 \mathrm{~min}\right)$ were counted in an automated Cell Counter (Invitrogen; Thermo Fisher Scientific, Inc.); 3 slides were counted in each group, with 3 fields of view from each.

Cell proliferation and DNA synthesis. Cell proliferation was determined using a CCK-8 assay, according to the manufacturer's instructions. Briefly, following the indicated treatment, $10 \mu 1$ of CCK-8 solution was added to each well, and the plate was incubated at $37^{\circ} \mathrm{C}$ for $2.5 \mathrm{~h}$. The color intensity was read at a wave length of $450 \mathrm{~nm}$. VSMC DNA synthesis was assessed by measuring the incorporation of BrdU. BrdU incorporation was measured using a cell proliferation ELISA kit according to the manufacturer's protocol. The effect of PAE on cell proliferation and DNA synthesis was expressed as the percentage compared with the control group, which was set at $100 \%$.

Flow cytometry analysis of cell cycle progression. Cell cycle progression was determined using PI staining according to the manufacturer's instructions and fluorescence-activated cellsorting (FACS). Following $24 \mathrm{~h}$ treatment with $200 \mu \mathrm{M}$ PAE and/or $20 \mathrm{ng} / \mathrm{ml}$ PDGF-BB, cells were harvested and fixed with $70 \%$ ethanol over night at $4^{\circ} \mathrm{C}$. Fixed cells were collected by centrifugation $\left(2,067 \mathrm{x} \mathrm{g}\right.$ at $4^{\circ} \mathrm{C}$ for $\left.5 \mathrm{~min}\right)$, washed once using PBS, and incubated with $1 \mathrm{ml}$ of PI staining buffer $(20 \mu \mathrm{g} / \mathrm{ml} \mathrm{PI}$ and $50 \mu \mathrm{g} / \mathrm{ml} \mathrm{RNaseA}$ ) at $4^{\circ} \mathrm{C}$ for $30 \mathrm{~min}$. Afterward, cellular fluorescence was measured by flow cytometry analysis with a FACS Calibur Flow Cytometer (BD Biosciences, Franklin Lakes, NJ, USA). Cell cycle distributions were analyzed using the Multi cycle AV software version 3 (Phoenix Flow Systems, San Diego, CA, USA).

Reverse transcription-quantitative polymerase chain reaction $(R T-q P C R)$. Total RNA was isolated from VSMCs using TRIzol reagent, according to the manufacturer's protocol. The RNA yields and purities were spectrophotometrically analyzed by the A260/A280 and A230/A260 ratios using a Nano Drop 2000c (Thermo Fisher Scientific, Inc., Wilmington, DE, USA). The RNA ( $2 \mu \mathrm{g}$ of each sample) was reverse transcribed into cDNA using oligo (dT) primers and the Transcript or First Strand cDNA Synthesis kit (cat. no. 04896866001; Roche Diagnostics) under the following conditions: $50^{\circ} \mathrm{C} 60 \mathrm{~min}$, $90^{\circ} \mathrm{C} 5 \mathrm{~min}$ and $4^{\circ} \mathrm{C} 90 \mathrm{~min}$. SYBR-Green PCR Master Mix (cat. no. 04707516001; Roche Diagnostics) was then used to quantify PCR amplifications with the Light Cycler 480 instrument (software version 1.5; Roche Diagnostics). The PCR 
conditions were as follows: Initial denaturation at $94^{\circ} \mathrm{C}$ for 2 min, followed by 25-35 amplification cycles consisting of denaturation at $94^{\circ} \mathrm{C}$ for $40 \mathrm{sec}$, annealing at $58^{\circ} \mathrm{C}$ for $45 \mathrm{sec}$ and elongation at $72^{\circ} \mathrm{C}$ for $1 \mathrm{~min}$. The following primer pairs were used for qPCR: OPN, forward 5'-GCTGAAGCCTGA CCCATC-3' and reverse 5'-TCCCGTTGCTGTCCTGAT-3'; cyclin dependent kinase (CDK) 4, forward 5'-ATGTTGTCC GGCTGATGG-3' and reverse 5'-CACCAGGGTTACCTT GATCTCC-3'; CDK2, forward 5'-GCTTTCTGCCATTCT CATCG-3' and reverse 5'-GTCCCCAGAGTCCGAAAG AT-3'; cyclin D1, forward 5'-CCCGAGGAGTTGCTGCAA ATGGA-3' and reverse 5'-AGGGCCACAAAGGTCTGT GCA-3'; cyclin E, forward 5'-GTCCTGGCTGAATGTATA CATGC-3' and reverse 5'-CCCTATTTTGTTCAGACAACA TGGC-3'; cyclin dependent kinase inhibitor 1A (CDKN1A), forward 5'-TGTACCAGCCACAGGCACCATGT-3' and reverse 5'-TCGCCATGAGCGCATCGCAAT-3'; GAPDH, forward 5'-GACATGCCGCCTGGAGAAAC-3', and reverse 5'-AGCCCAGGATGCCCTTTAGT-3'. The $2^{-\Delta \Delta C q}$ method was used to analyze data from real-time quantitative PCR experiments (13).

Scratch migration test. VSMCs were plated in 6-well plates, grown to confluence to forma monolayer and treated with PDGF-BB and/or PAE for $24 \mathrm{~h}$. Subsequently, the cells were serum-starved for $4 \mathrm{~h}$. Wounds were created by scraping the cell monolayer with a $200 \mu 1$ sterile micropipette tip and rinsing 3 times with PBS. All of the cells were stimulated with basal medium (DMEM/F12 containing $0.5 \%$ FBS and $0.5 \%$ PS). At $0,6,12$, and 24 h post-injury, migratory cells were photo graphed using an inverted microscope (IX51; Olympus Corporation, Tokyo, Japan).

ROS measurement. Intracellular ROS generation was determined by 2',7'-dichlorofluorescin diacetate (DCFH-DA; cat. no. D6883; Sigma-Aldrich; Merck KGaA) which is oxidized to fluorescent DCF by ROS. Following $1 \mathrm{~h}$ pre treatment with PAE, cells were incubated with PDGF-BB for $2 \mathrm{~h}$. Subsequently, VSMC cells were washed twice and incubated with $5 \mu \mathrm{M}$ of DCFH-DA solution in serum-free medium at $37^{\circ} \mathrm{C}$ for $30 \mathrm{~min}$ in the dark. Data were collected using a fluorescence readeratan excitation/emission wave length of 488 and $525 \mathrm{~nm}$. A fluorescence microscope (cat. no. IX51; Olympus Corporation) was also used to evaluate the DCF fluorescence of cells on cover slip.

Western blotting analysis. Protein was extracted from VSMCs indifferent groups. The cells were lysed in RIPA lysis buffer (Wuhan Goodbio Technology Co. Ltd., Wuhan, China) containing $50 \mathrm{mM}$ Tris- $\mathrm{HCl}, 150 \mathrm{mM} \mathrm{NaCl}, 1 \%$ Triton $\mathrm{X}-100$, $1 \%$ sodium deoxycholate and $0.1 \%$ SDS; the cells were then scraped into $1.5-\mathrm{ml}$ centrifuge tubes. The cell suspension was centrifuged at $3,362 \times \mathrm{g}$ for $30 \mathrm{~min}$ at $4^{\circ} \mathrm{C}$ and the protein concentration was measured using a bicinchoninic acid protein assay kit (23227; Thermo Fisher Scientific, Inc.) using the Synergy HT microplate reader. The cell lysates $(40 \mu \mathrm{g}$ per lane) were separated by $10 \%$ SDS-PAGE, then proteins were transferred on to a polyvinylidene fluoride membrane and incubated with the primary anti bodies diluted 1:1,000 in $5 \% \mathrm{w} / \mathrm{v}$ non-fat dry milk, $1 \mathrm{X}$ Tris-buffered saline, $0.1 \%$

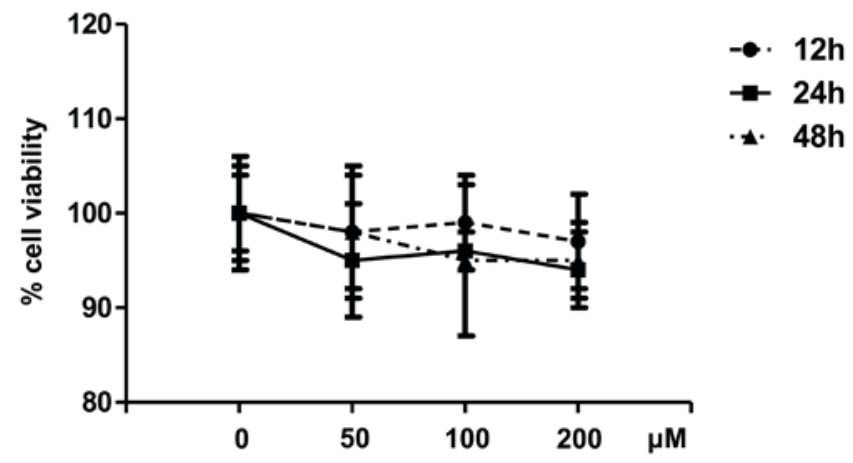

Figure 1. Effect of PAE on cell viability. Cell viability was examined by the trypan blue exclusion test following 12,24 and $48 \mathrm{~h}$ of treatment with 50, 100 and $200 \mu \mathrm{M}$ PAE. Data are expressed as the mean \pm standard deviation of 5 independent replicates. PAE, paeoniflorin.

Tween-20 at $4^{\circ} \mathrm{C}$ with gentle agitation, overnight. The primary antibodies were p-p38, p38, p-JNK, JNK, p-ERK1/2, ERK1/2 and GAPDH. There after, membranes were incubated with these condary antibody, goat anti-rabbit immunoglobulin $\mathrm{G}$ (926-32211; 1:100; LI-COR Biosciences) for $60 \mathrm{~min}$. The blots were scanned by an Odyssey two-color infrared imaging system (version 3.0; LI-COR Biosciences) to quantify protein expression. Protein expression levels were normalized to the level of GAPDH expression.

Statistical analysis. All data are expressed as the mean \pm standard deviation of $\geq 3$ replicates and analyzed using SPSS version 19.0 (IBM Corp., Armonk, NY, USA). Comparisons of two groups were performed using the unpaired Student's t test. Multiple comparison analyses were performed by one-way analysis of variance, followed by Student-Newman-Keuls tests. $\mathrm{P}<0.05$ was considered to indicate a statistically significant difference.

\section{Results}

PAE does not affect the viability of VSMCs. The cytotoxicity of PAE on VSMCs was assessed using the trypan blue exclusion test. Compared with untreated cells, concentrations of 50, 100 , and $200 \mu \mathrm{M}$ PAE did not result in significant cytotoxicity in VSMCs following 12, 24 or $48 \mathrm{~h}$ of incubation (Fig. 1). The cell viability in different groups was all maintained at $\sim 94 \%$, there by indicating that PAE had no cytotoxicity at the tested concentrations.

PAE inhibits VSMC proliferation and DNA synthesis induced by $P D G F-B B$. To determine the effect of PAE on VSMC proliferation, CCK-8 cell proliferation assays were performed on VSMCs treated with different concentrations of PAE $(50,100$, and $200 \mu \mathrm{M})$ and PDGF-BB $(20 \mathrm{ng} / \mathrm{ml})$ for 12, 24 or 48 h. Compared with the control group, PDGF-BB significantly induced VSMC proliferation in a time-dependent manner, which was blocked by PAE in a time-and concentration-dependent manner; the most remarkable suppression of proliferation compared with PDGF-BB only was obtained at $200 \mu \mathrm{M}$ (Fig. 2A). The suppressive effect of PAE on DNA 

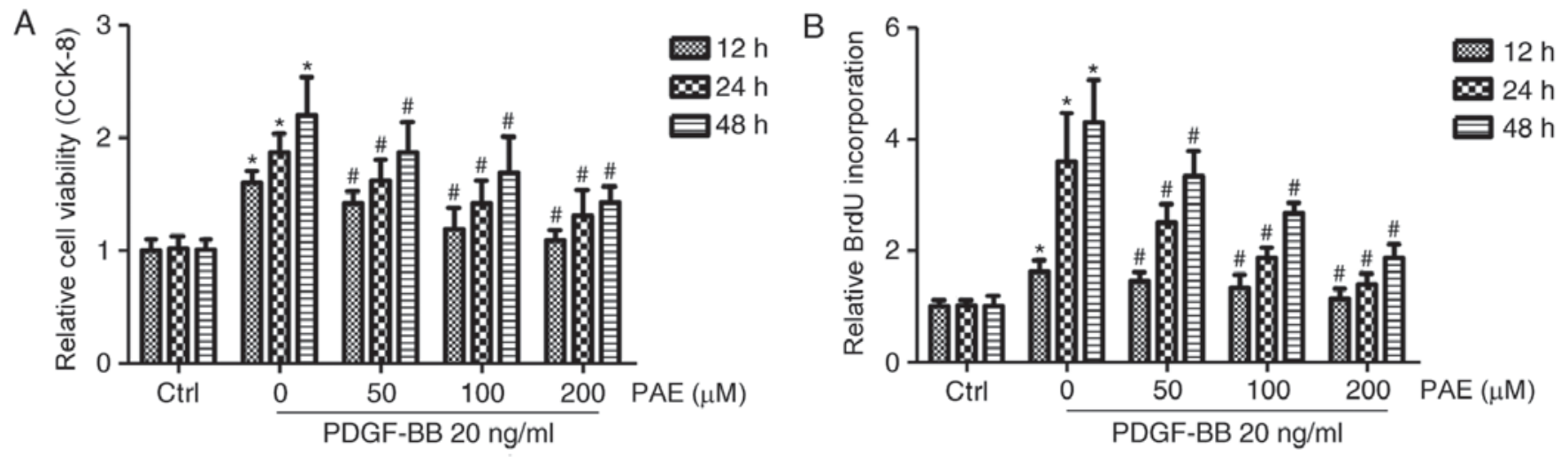

Figure 2. Effect of PAE on the proliferation of VSMCs. VSMCs were pre-cultured inserum-free medium for $24 \mathrm{~h}$ and then incubated in the absence or presence of PAE $(50,100,200 \mu \mathrm{M})$ with $20 \mathrm{ng} / \mathrm{ml}$ PDGF-BB for a further 12, 24 or $48 \mathrm{~h}$. (A) Cell proliferation was examined by the CCK-8 test. (B) DNA synthesis was determined with an ELISA-based BrdU incorporation assay. Data are expressed as the mean \pm standard deviation of 5 independent replicates. ${ }^{*} \mathrm{P}<0.05$ vs. control; " $\mathrm{P}<0.05$ vs. PDGF-BB only. PAE, paeoniflorin; VSMCs, vascular smooth muscle cells; PDGF-BB, platelet derived growth factor-BB; CCK-8, Cell Counting Kit-8.
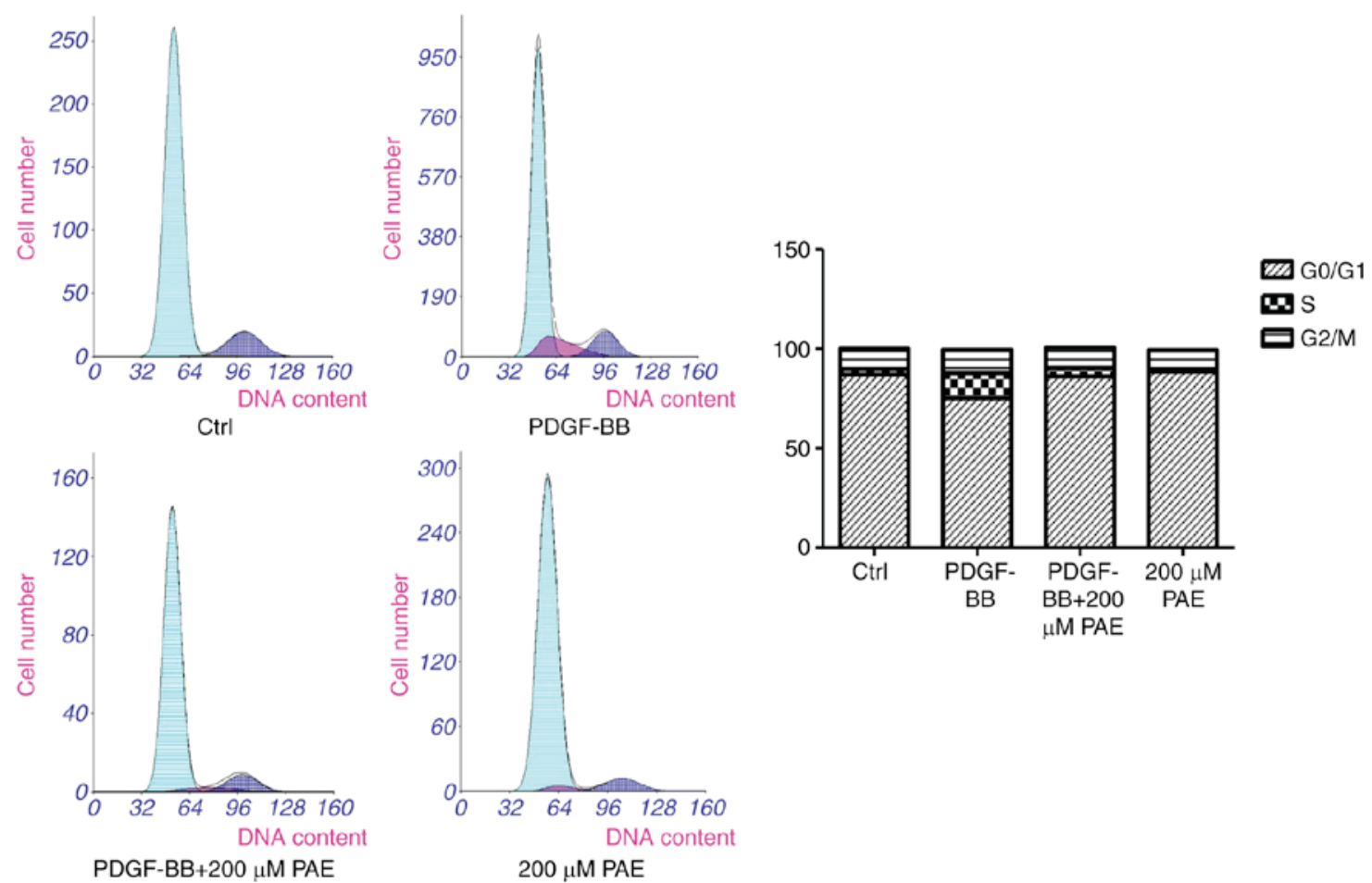

Figure 3. Effect of PAE on cell cycle progression. VSMCs were grown with $200 \mu \mathrm{M}$ PAE in the absence or presence of $20 \mathrm{ng} / \mathrm{ml}$ PDGF-BB for $24 \mathrm{~h}$. Flow cytometric analysis of propidium iodide fluorescence in VSMCs was used to determine the number of VSMCs in the G0/G1, S and G2/M phases. Each experiment was performed 3 independent times. PAE, paeoniflorin; VSMCs, vascular smooth muscle cells; PDGF-BB, platelet derived growth factor-BB.

synthesis was then investigated by measuring the incorporation of BrdU. Compared with the control group, PDGF-BB significantly increased DNA synthesis in VSMCs in a time-dependent manner, which was also blocked by PAE in a concentration-and time-dependent manner (Fig. 2B).

PAE blocks PDGF-BB-induced cell cycle progression through arrest of G0/G1 to Sphasetransition. Flow cytometry analysis was used to analyze the effect of PAE on cell cycle progression. PDGF-BB was demonstrated to increase the percentage of cells in Sphase, where as the populations of G0/G1 were decreased, compared with control (Fig. 3).
This condition was reversed when the cells were treated with $200 \mu \mathrm{M}$ PAE, there by indicating that PAE suppressed cell cycle progression; in addition, $200 \mu \mathrm{M}$ PAE increased the G0/G1 populations and depressed the percentage of cells in Sphase compared with the PDGF-BB group. These results therefore demonstrated that PAE affected the G0/G1 to Sphase transition rather than being involved in the $\mathrm{S}$ or $\mathrm{G} 2 / \mathrm{M}$ phase (Fig. 3).

Effect of PAE on mRNA expression levels of cell cycle regulatory and migration genes and cell migration. To characterize the potential mechanism responsible for PAE-induced cell 

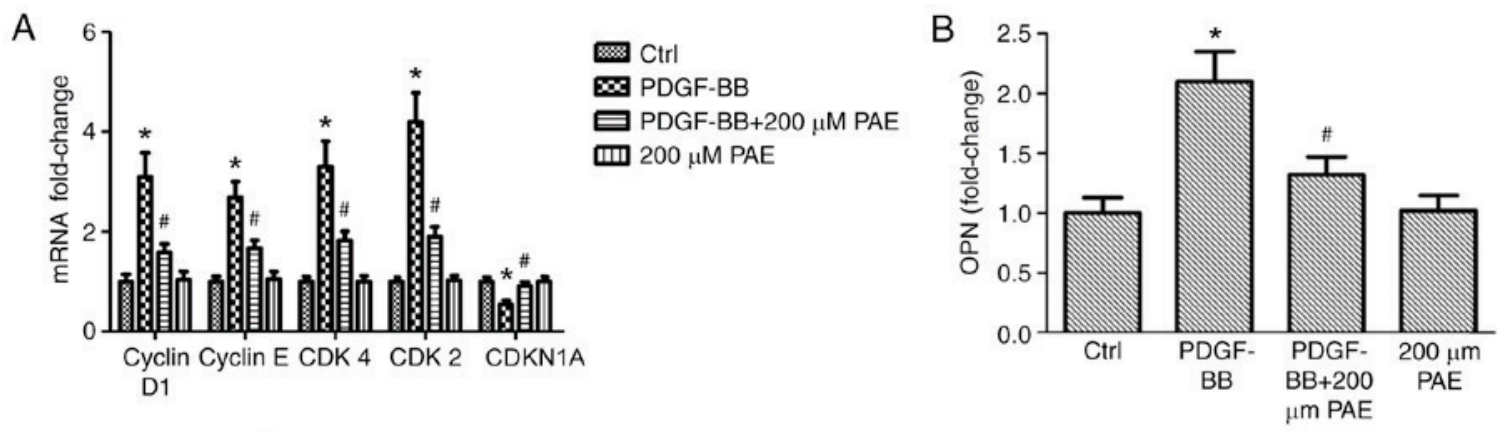

C
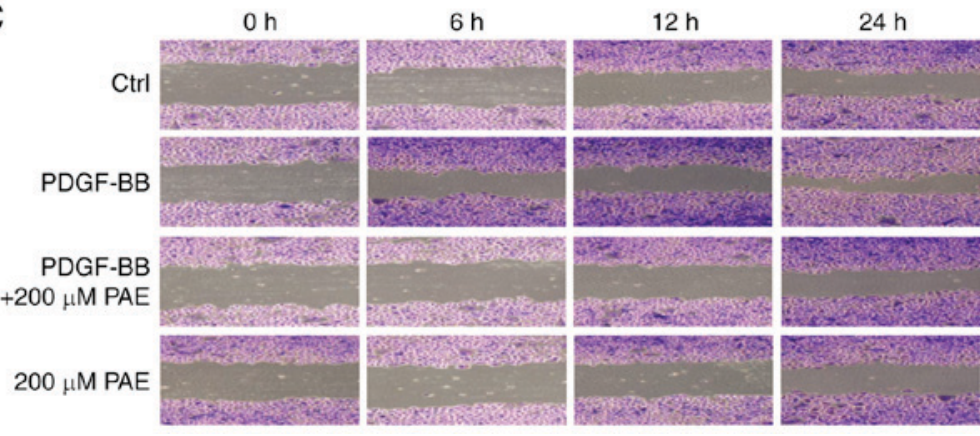

Figure 4. Effect of PAE on the mRNA expression levels of cell cycle regulatory and migration genes and cell migration in VSMCs. VSMCs were grown with PAE $(200 \mu \mathrm{M})$ in the absence or presence of PDGF-BB $(20 \mathrm{ng} / \mathrm{ml})$ for $24 \mathrm{~h}$, then mRNA expression levels of (A) cyclin D1, cyclin E, CDK4, CDK2, CDKN1A and (B) OPN were analyzed by reverse transcription-quantitative polymerase chain reaction. Data are expressed as the mean + standard deviation of 3 independent replicates. "P $<0.05$ vs. control; ${ }^{*} \mathrm{P}<0.05$ vs. PDGF-BB only. (C) In vitro scratch migration assay, in which cells were treated with $200 \mu \mathrm{M} P A \mathrm{E}$ in the absence or presence of $20 \mathrm{ng} / \mathrm{ml}$ PDGF-BB and imaged at 0, 6, 12 and $24 \mathrm{~h}$. Each experiment was performed 3 independent times. PAE, paeoniflorin; VSMCs, vascular smooth muscle cells; PDGF-BB, platelet derived growth factor-BB; CDK, cyclin dependent kinase; CDKN1A, cyclin dependent kinase inhibitor 1A; OPN, osteopontin.
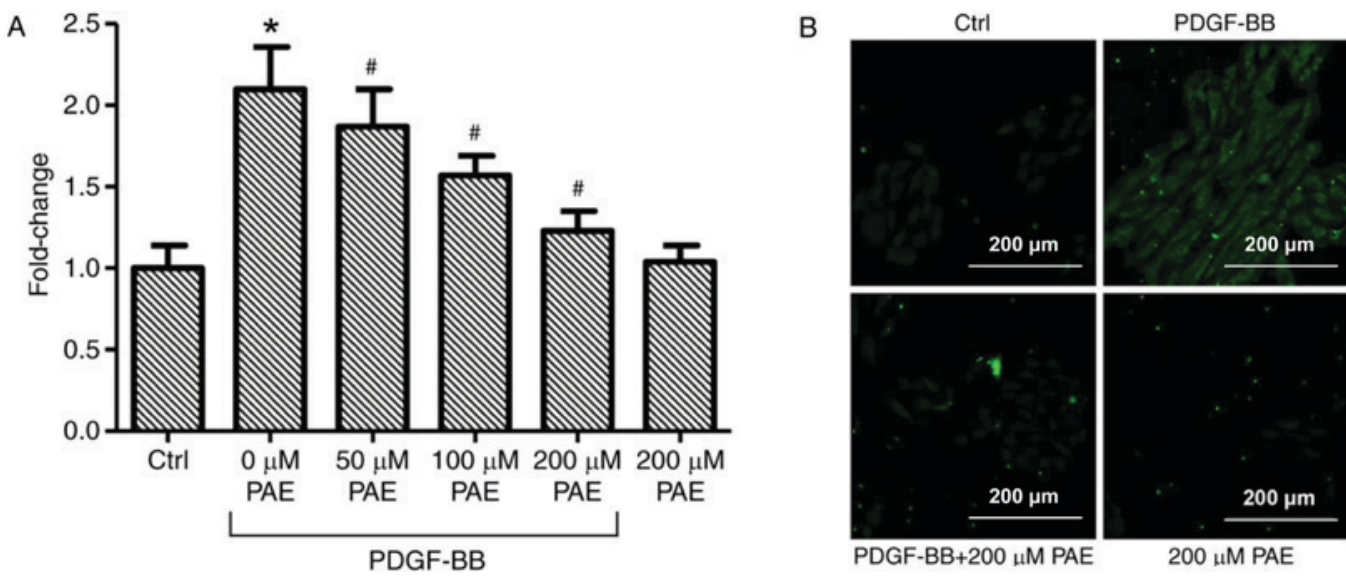

Figure 5. PAE inhibits ROS generation induced by PDGF-BB. ROS production was assessed by (A) quantitative assay and (B) fluorescence microscopy. ${ }^{*} \mathrm{P}<0.05$ vs. control; ${ }^{\#} \mathrm{P}<0.05$ vs. PDGF-BB only. Each experiment was performed 3 independent times. PAE, paeoniflorin; ROS, reactive oxygen species; PDGF-BB, platelet derived growth factor-BB.

cycle arrest and cell migration events, RT-qPCR was used to examine expression of cyclins, CDKs, CDKN1A and the cell cycle inhibitory expression and cell migration-related protein, OPN. Compared with the control group, PDGF-BB treatment significantly increased the expression levels of genes related to cell cycle, including cyclin D1, cyclin E, CDK4 and CDK2 (Fig. 4A), and expression of OPN, which is related to cell migration (Fig. 4B). However, mRNA expression of CDKN1A was down regulated significantly compared with control (Fig. 4A). PAE down regulated PDGF-BB-induced expression of CDK4, $\mathrm{CDK} 2$, cyclin D1, cyclin $\mathrm{E}$ and OPN genes, but up regulated the expression of CDKN1A (Fig. 4A and B). Never the less, the use of PAE alone had no significant effect on gene expression (Fig. 4A and B). The effect of PAE on cell migration was also detected by scratch assay; cells stimulated with PDGF-BB migrated faster than the basal medium-treated cells, and PAE visibly inhibited cell migration (Fig. 4C).

PAE decreases PDGF-BB-induced production of ROS. VSMCs which were incubated with DCFH-DA demonstrated increased fluorescence intensity when treated with PDGF-BB compared with control, which indicated that PDGF-BB induced ROS 
A

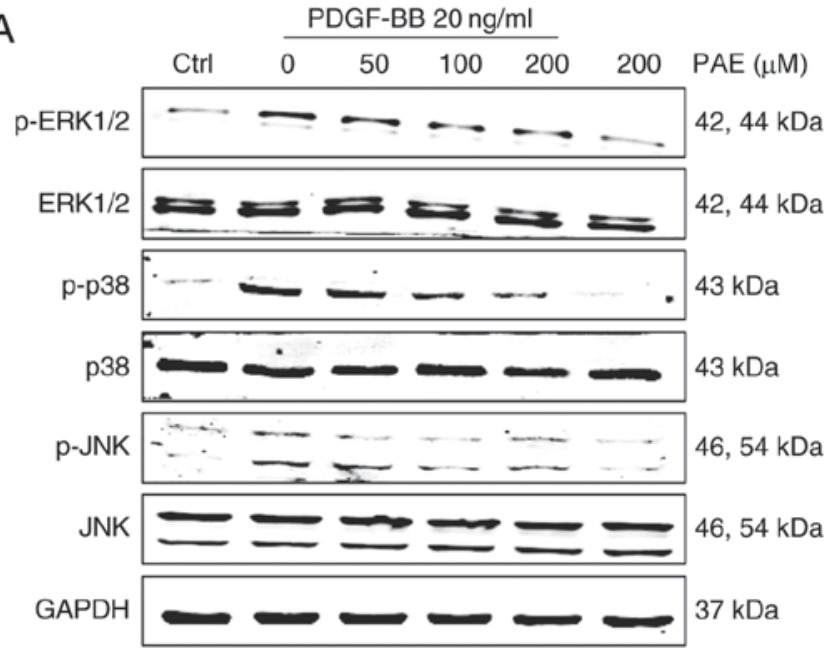

B

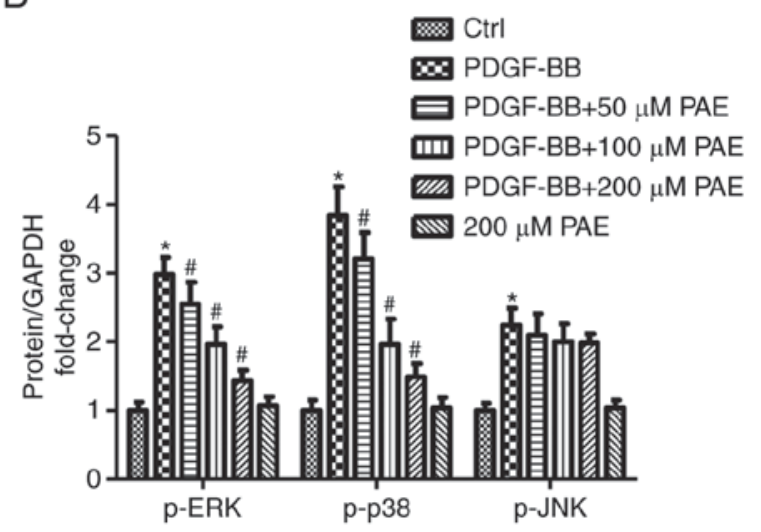

Figure 6. Effect of PAE on the activation of mitogen-activated protein kinase signalling pathways in PDGF-BB-induced VSMCs. VSMCs were pre treated with PAE $(50,100,200 \mu \mathrm{M})$ for $1 \mathrm{~h}$ prior to $20 \mathrm{ng} / \mathrm{ml}$ PDGF-BB treatment. The phosphorylation of p38, ERK, JNK induced by 15 min of PDGF-BB treatments was analyzed by western blotting analysis. (A) Representative western blots and (B) quantitative analysis. Data are expressed as the mean + standard deviation of $\geq 3$ independent replicates. ${ }^{*} \mathrm{P}<0.05$ vs. control; ${ }^{~} \mathrm{P}<0.05$ vs. PDGF-BB only. PAE, paeoniflorin; PDGF-BB, platelet derived growth factor-BB; VSMCs, vascular smooth muscle cells; p-, phosphorylated; ERK1/2, extracellular signal-related protein kinases 1 and 2; JNK, c-Jun N-terminal kinase.

production (Fig. 5A and B). PAE treatment significantly reduced PDGF-BB-induced ROS production in a concentration-dependent manner compared with PDGF-BB only (Fig. 5A and B). Microscopic examination of DCF-derived fluorescence, also revealed that that $200 \mu \mathrm{M}$ PAE visibly inhibited the accumulation of intra cellular ROS in PDGF-BB-treated cells compared with PDGF-BB alone (Fig. 5B). In addition, no effect on ROS generation was observed in cells treated with $200 \mu \mathrm{M}$ PAE alone compared with control cells (Fig. 5A and B).

Molecular mechanisms involved in the inhibition of VSMC proliferation by PAE. To explore the molecular mechanisms by which PAE inhibited VSMC proliferation, the effects of PAE on MAPK signalling pathway activation were examined. Significant activation of ERK1/2, p38, and JNK was observed following $15 \mathrm{~min}$ of PDGF-BB treatment compared with control, but the total levels of these molecules were not affected (Fig. 6). Compared with PDGF-BB only, PAE significantly reduced the phosphorylation of p38 and ERK1/2 in a concentration-dependent manner, but exhibited no inhibitory effect on the phosphorylation of JNK (Fig. 6).

\section{Discussion}

In the present study, VSMC proliferation and migration was demonstrated to be enhanced by PDGF-BB compared with control, and inhibited by PAE compared with PDGF-BB alone in a concentration-dependent manner, without cell cytotoxicity. PAE suppressed the cell cycle at the G0/G1 to Sphase by inhibiting them RNA expression of cyclin D1, cyclin E, CDK4, and CDK2; this glycoside also increased them RNA expression of CDKN1A in PDGF-BB-stimulated VSMCs. PDGF-BB-induced expression of OPN was also prevented by PAE supplementation. These beneficial effects of PAE on the proliferation of VSMCs were associated with the inhibition of ROS production, and reduced activation of the ERK1/2 and p38 signaling pathways.
PAE inhibits the proliferation of lung cancer cells $(14,15)$, HeLa human cervical cancer cells (16) and human colorectal carcinoma (HT29) cells by blocking cell cycle progression in the G0/G1 or Sphase and inducing apoptosis. However, the effect of PAE on PDGF-BB-induced proliferation of VSMCs remains unclear. The present study demonstrated for the first time, to the best of our knowledge, that PAE inhibited PDGF-BB-induced proliferation of VSMCs. According to previous research, VSMCs change from a contractile phenotype to proliferative phenotype in the presence of PDGF-BB (17). Never the less, the combined use of PAE and PDGF-BB would inhibit such a change in phenotype. The results of CCK-8 and BrdU assays indicated that PAE inhibited PDGF-BB-induced cell proliferation. Flow cytometry demonstrated that PAE arrested the cells in transition from G0/G1 phase to Sphase. Expression of genes that are involved in the check points of the transition from G0/G1 phase to Sphase were also examined: PDGF-BB considerably upregulated them RNA expression levels of cyclin D1, cyclin E, CDK4 and CDK2, but down regulated them RNA expression of CDKN1A. The combined use of PAE and PDGF-BB reversed the changes in mRNA expression levels of these genes. Thus PAE antagonized PDGF-BB-induced cell proliferation by regulating the cell cycle-related proteins.

In atherosclerosis and restenosis following percutaneous coronary intervention, the migration of VSMCs is an important step in intimal formation (18). OPN is the marker gene for the change of VSMCs from a contractile to proliferative phenotype. The expression of OPN is closely associated with the migration ability of VSMCs $(19,20)$. The present study demonstrated that following stimulation with PDGF-BB for $48 \mathrm{~h}$, OPN mRNA expression increased, which corresponded to strong cell migration ability. However, the combined use with PAE reversed this phenomenon.

ROS are important for the proliferation and migration of VSMCs. Previous cell experiments indicated that a large amount of ROS are conducive to the proliferation of VSMCs 
and to intimal hyperplasia (21). In the present study, PAE was demonstrated to significantly inhibit PDGF-BB-induced generation of ROS, there by indicating that the inhibitory effect of PAE on the proliferation of VSMCs may be partially related to the inhibition of ROS generation. Further more, the activation levels of ROS-induced down stream MAPK signalling pathways were examined. MAPK signalling consists of a sequence of successively functioning kinases that ultimately result in the dual phosphorylation and activation of $\mathrm{p} 38, \mathrm{JNK} 1 / 2$ and ERK1/2. MAPK signalling pathways are be activated during PDGF-BB-induced proliferation of VSMCs $(4,5)$. ERK and p38 MAPK signalling pathways have important regulatory functions in vascular reconstruction (22). According to the results of the present study, PAE significantly inhibited PDGF-BB-induced activation of ERK and p38 MAPK signalling pathways, but had little impact on JNK signaling. Therefore, PAE was found to inhibit PDGF-BB-induced proliferation and migration of VSMCs by inhibiting the ERK and p38 MAPK signalling pathways.

In summary, to the best of our knowledge, the present study observed for the first time that PAE inhibited PDGF-BB-induced proliferation and migration of VSMCs. PAE may regulate cell cycle and expression level of cell migration-related proteins by inhibiting ROS-mediated ERK1/2 and p38 signaling pathways. PAE may also serve as a potential drug against atherosclerosis and restenosis following percutaneous coronary intervention. However, the findings of the present experiment should be confirmed by experiments in large animals.

\section{References}

1. Ding Z, Liu S, Wang X, Deng X, Fan Y, Sun C, Wang Y and Mehta JL: Hemodynamic shear stress via ROS modulates PCSK9 expression in human vascular endothelial and smooth muscle cells and along the mouse aorta. Antioxid Redox Signal 22: 760-771, 2015.

2. Sadowitz B, Seymour K, Gahtan V and Maier KG: The role of hyaluronic acid in atherosclerosis and intimal hyperplasia. J Surg Res 173: e63-e72, 2012.

3. Rivard A and Andres V: Vascular smooth muscle cell proliferation in the pathogenesis of atherosclerotic cardio vascular diseases. Histol Histopathol 15: 557-571, 2000.

4. Chang Y, Uen YH, Chen CC, Lin SC, Tseng SY, Wang YH, Sheu JR and Hsieh CY: Platonin inhibited PDGF-BB-induced proliferation of rat vascular smooth muscle cells via JNK1/2-dependent signaling. Acta Pharmacol Sin 32: 1337-1344, 2011.

5. Wang Y, Wang Y, Liu D, Wang W, Zhao H, Wang M and Yin H: Cordyceps sinensis polysaccharide inhibits PDGF-BB-induced inflammation and ROS production in human mesangial cells Carbohydr Polym 125: 135-145, 2015.

6. Lee CK, Lee HM, Kim HJ, Park HJ, Won KJ, Roh HY, Choi WS, Jeon BH, Park TK and Kim B: Syk contributes to PDGF-BB-mediated migration of rat aortic smooth muscle cells via MAPK pathways. Cardiovasc Res 74: 159-168, 2007.

7. Lee KP, Lee K, Park WH, Kim H and Hong H: Piperine inhibits platelet-derived growth factor-BB-induced proliferation and migration in vascular smooth muscle cells. J Med Food 18: 208-215, 2015.
8. Zhou H, Yang HX, Yuan Y, Deng W, Zhang JY, Bian ZY, Zong J, Dai J and Tang QZ: Paeoniflorin attenuates pressure overload-induced cardiac remodeling via inhibition of TGF $\beta /$ Smads and NF- $\kappa$ B pathways. J Mol Histol 44: 357-367, 2013.

9. Chen C, Du P and Wang J: Paeoniflorin ameliorates acute myocardial infarction of rats by inhibiting inflammation and inducible nitric oxide synthase signaling pathways. Mol Med Rep 12: 3937-3943, 2015.

10. Cao W, Zhang W, Liu J, Wang Y, Peng X, Lu D, Qi R, Wang Y and Wang H: Paeoniflorin improves survival in LPS-challenged mice through the suppression of TNF-alpha and IL-1 beta release and augmentation of IL-10 production. Int Immunopharmacol 11: 172-178, 2011.

11. Nizamutdinova IT, Jin YC, Kim JS, Yean MH, Kang SS, Kim YS, Lee JH, Seo HG, Kim HJ and Chang KC: Paeonol and paeoniflorin, the main active principles of Paeonia albiflora, protect the heart from myocardial ischemia/reperfusion injury in rats. Planta Med 74: 14-18, 2008.

12. Chen C, Tang Y, Deng W, Huang C and Wu T: Salidroside blocks the proliferation of pulmonary artery smooth muscle cells induced by platelet-deried growth factor-BB. Mol Med Rep 10: 917-922, 2014.

13. Livak KJ and Schmittgen TD: Analysis of relative gene expression data using real-time quantitative PCR and the 2(-Delta Delta C(T)) method. Methods 25: 402-408, 2001.

14. Hung JY, Yang CJ, Tsai YM, Huang HW and Huang MS: Antiproliferative activity of paeoniflorin is through cell cycle arrest and the Fas/Faslig and-mediated apoptotic pathway in human non-small cell lung cancer A549 cells. Clin Exp Pharmacol Physiol 35: 141-147, 2008.

15. Wu Q, Chen GL, Li YJ, Chen Y and Lin FZ: Paeoniflorin inhibits macrophage-mediated lung cancer metastasis. Chin J Nat Med 13: 925-932, 2015.

16. Zhang L and Zhang S: Modulating Bcl-2 family proteins and caspase-3 in induction of apoptosis by paeoniflorin in human cervical cancer cells. Phytother Res 25: 1551-1557, 2011.

17. Wang H, Zhou H, Wang CX, Li YS, Xie HY, Luo JD and Zhou Y: Paeoniflorin inhibits growth of human colorectal carcinoma HT29 cells in vitro and in vivo. Food Chem Toxicol 50: 1560-1567, 2012

18. Karki R, Kim SB and Kim DW: Magnolol inhibits migration of vascular smooth muscle cells via cytoskeletal remodeling pathway to attenuate neointima formation. Exp Cell Res 319: 3238-3250, 2013

19. Jiang H, Lun Y, Wu X, Xia Q, Zhang X, Xin S and Zhang J: Association between the hypomethylation of osteopontin and integrin $\beta 3$ promoters and vascular smooth muscle cell phenotype switching in great saphenous varicose veins. Int J Mol Sci 15: 18747-18761, 2014.

20. Chen JJ, Zhang J, Cai Y, Zhou YB, Wen GB, Tang CS, Qi YF and Jiang ZS: C-type natriuretic peptide inhibiting vascular calcification might involve decreasing bone morphogenic protein 2 and osteopontin levels. Mol Cell Biochem 392: 65-76, 2014.

21. Rodrigues E, Mariutti LR and Mercadante AZ: Scavenging capacity of marine carotenoids against reactive oxygen and nitrogen species in a membrane-mimicking system. Mar Drugs 10: 1784-1798, 2012.

22. Muslin AJ: MAPK signalling in cardio vascular health and disease: Molecular mechanisms and therapeutic targets. Clin Sci (Lond) 115: 203-218, 2008.

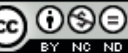

This work is licensed under a Creative Commons Attribution-NonCommercial-NoDerivatives 4.0 International (CC BY-NC-ND 4.0) License. 